\title{
Binary star speckle measurements at Calar Alto. I.
}

\author{
J. A. Docoboํ, V. S. Tamazian¹, Yu. Yu. Balega ${ }^{2}$, J. Blanco ${ }^{1}$, A. F. Maximov², and V. A. Vasyuk ${ }^{2}$ \\ 1 Astronomical Observatory Ramon Maria Aller, University of Santiago de Compostela. PO Box 197, \\ Santiago de Compostela, Spain \\ 2 Special Astrophysical Observatory, Zelenchuk region, 369167, Russia \\ Received 24 May 2000 / Accepted 14 November 2000
}

\begin{abstract}
We present the first results of our speckle interferometric measurements of binary stars made with the ICCD speckle camera using the 1.52-m telescope of the Observatorio Astronomico Nacional at Calar Alto (Spain) in September, 1999. The data contain 123 observations of 83 systems. The measured angular separations range from $0^{\prime \prime} 153$ to $6{ }^{\prime \prime} 727$. We have used there new speckle measurements to improve the orbital elements for the binaries COU 247 and BU $524 \mathrm{AB}$.
\end{abstract}

Key words. binaries: visual - interferometry

\section{Introduction}

At the present time, although the orbits for more than 1000 visual binaries are available, the study of orbital motion of visual and interferometric pairs remains an important astronomical discipline. Visual binaries are the key source of information about stellar masses and distances, while for the lower part of the main sequence they define practically our understanding of stellar physical properties. However, visual measurements of binaries with a filar micrometer are rarely performed today. In comparison with digital speckle interferometric methods, micrometric data show, in general, lower precision. They are also often associated with significant errors, which is especially true for small angular separations, $\sim 0$ '! 1 .

By means of speckle interferometry, the Rayleigh resolution limit of 70 mas $(0.07$ arcsec $)$ for a $1.5-\mathrm{m}$ telescope can be routinely achieved. That is high enough to study the relative motion of the components of the most well known visual binary stars, in order to define the parameters of their orbits. Only for very close visual pairs are the larger apertures needed, but the lack of the observing time at large telescopes is the reason for rare and unsystematic binary star observations.

Therefore, high quality speckle measurements, even with moderate size telescopes, can be an important source of data about the relative motion of the components in multiple star systems (Douglass et al. 1997). For these purposes a speckle interferometer with a photoncounting intensified CCD detector was developed in the Astronomical Observatory Ramon Maria Aller of the University of Santiago de Compostela, in cooperation with the Special Astrophysical Observatory (SAO) of the

Send offprint requests to: J. A. Docobo
Russian Academy of Sciences. The new instrument has been used for speckle observations of binary stars using the 1.52-m telescope at Calar Alto, in 1999. Below, we provide a brief description of the instrument and report on the first interferometric data obtained. Orbits of visual binaries Cou 247 and $\mathrm{Bu} 524$ were improved using the new measurements.

\section{Brief description of the speckle camera}

The scheme of the instrument is essentially the same as for other speckle cameras in use today at several large telescopes. The main module contains a pair of interchangeable microscope objectives with magnifications $8 \times$ and $20 \times$, which are necessary to sample the size of individual speckles (about $4 \mu$ at $500 \mathrm{~nm}$ at the $\mathrm{f} / 8$ Cassegrain focus of the 1.52-m telescope) to a detector's pixel, with a size of $13.4 \mu$. The corresponding scale on the detector is 0.028 or 0.011 arcsec per pixel with total fields of view of 5.6 and 14.3 arcsec square. We normally use a $20 \times$ microscope objective; the $8 \times$ one is intended only for pointing at weak stars. In front of the microscope objective is the Uniblitz remote-controlled electronic shutter, which provides the exposure value in the range 5 to $40 \mathrm{~ms}$. The shutter is synchronized with the CCD detector readout. An additional mirror, which can be installed in the beam in front of the shutter, sends light to the TV guidance camera for centering of an object in the field of view. A combination of narrow-pass band interference filters and neutral density filters is used for wavelength selection. Data are routinely obtained through the 520/24-nm filter; however, a filter wheel assembly also includes 600/50-nm and 660/40-nm filters. A set of 4 zero mean deviation prisms, mounted on a rotation stage, is used for atmospheric dispersion compensation at different zenith angles. Each prism is 
designed to compensate for the atmospheric effect within a prescribed interval of zenith angle, while for the $20^{\circ}$ radius from the zenith, the clear aperture is selected. The detector system consists of a PCO Computer Optics (Germany) Sensicam CCD camera with $1280(H) \times 1024(V)$ pixels of $6.7 \times 6.7 \mu \mathrm{m}$, optically coupled by means of a pair of $\mathrm{f} / 1.5$ transfer lenses to a 3 -stage electrostatically focused image intensifier. The input 24-mm photocathode of the intensifier has an S-25 spectral response with a peak sensitivity of $12 \%$ at $510 \mathrm{~nm}$, and about $2 \%$ sensitivity is still available at $800 \mathrm{~nm}$. For faster readout we use the sampling of speckle images to $512 \times 512$ pixels. The dynamic range of the system is limited by the 12-bit digitization. Single photoelectron events are recorded by the system with a signal-to-noise ratio of about 30 . Shorter exposures and narrower filters are utilized when bright stars are studied in an analogue readout mode. The data are transferred via optical fibers to a computer system and then onto Exabyte tapes. The system is capable of acquiring and storing 12-bit digitized data at a speed of 5 images per second.

\section{Observations and data reduction}

The speckle camera has been primarily used for observations of binary stars at the Cassegrain focus of the 1.52-m telescope of the Observatorio Astronomico Nacional at Calar Alto, Spain. 83 pairs were observed between September 15 and 24, 1999, under a moderate seeing of $1^{\prime \prime}$ to $3^{\prime \prime}$. For each binary, a typical observing procedure involved the accumulation of 1000 to 3000 short exposure images on Exabyte tapes.

Calibration of our speckle data was accomplished only by observing wide binaries with very long orbital periods. A list of 10 pairs observed for calibration purpose on the 6-m telescope of SAO was used to define the orientation of the CCD with respect to the vertical. In addition, the detector orientation was checked by using star trails in right ascension with the magnification $8 \times$. We could not use other calibration procedures because the telescope was not equipped with a slit mask and its focal length was not known to sufficient accuracy. The resulting value of the camera orientation error is less than $0.2^{\circ}$.

Processing of speckle data is made in three steps. Firstly, for each speckle frame we make a flat-field photometric correction and geometric correction of field distortions caused by the image intensifier. Then, we compute the mean power spectrum of an object following the standard Labeyrie procedure (1970). The average power spectrum is corrected for the photon noise bias. At the final stage, we compute a set of radial cross-sections through the power spectrum up to the diffraction cut-off frequency of the telescope and fit this with the model of a binary star spectrum to find the distance and position angle. From position measurements for pairs with at least 3 observations, we have found that the mean standard deviation in separation is 10 mas, while the error in position angle is $1^{\circ}$. With the present detector, we could observe binaries with
Table 1. Orbital elements for COU 247 and BU 524 AB

\begin{tabular}{lll}
\hline Star & COU 247 & BU 524 AB \\
Author(s) & Blanco J. & Docobo-Vasyuk \\
$P($ years $)$ & $280.00^{y}$ & $31.528^{y} \pm 0.072^{y}$ \\
$T$ & 1990.21 & $1996.526 \pm 0.014$ \\
$e$ & 0.426 & $0.753 \pm 0.005$ \\
$a$ & $0.846^{\prime \prime}$ & $0.221^{\prime \prime} \pm 0.003^{\prime \prime}$ \\
$i$ & $124.9^{\circ}$ & $121.0^{\circ} \pm 0.6^{\circ}$ \\
$\Omega$ & $21.3^{\circ}$ & $25.4^{\circ} \pm 0.6^{\circ}$ \\
$\omega$ & $47.1^{\circ}$ & $265.0^{\circ} \pm 0.6^{\circ}$ \\
$\Sigma$ masses & $2.9 M_{\odot}$ & $4.1 M_{\odot} \pm 0.4 M_{\odot}$ \\
\hline
\end{tabular}

a secondary as faint as 10.5 magnitude. However, we expect that with an improved image intensifier and by using the cooling of the photocathode, the limiting magnitude will be near 13 .

Results of the measurements are given in Cols. (1) to (6) of Table 2. The first three columns list the Washington Double Star Catalogue coordinate (Worley $\&$ Douglass 1996), the name of the star or its catalogue number in common use and the discoverer designation. The fourth column gives the epoch of the observation in fractional Besselian year. The fifth and sixth columns contain the measured position angle $\theta$ in degrees and angular separation $\rho$ in arcseconds. The note "UR" denotes the cases when the binary was not resolved. This might indicate too close a companion (less than 70 mas), too large a magnitude difference (more than 3 magnitudes), very bad seeing, or any combination of these factors. Finally, the note " $R$ " means the use of the red $660 / 40$-nm filter.

\section{New orbits}

Using the measurements presented in this paper, the orbits of the systems COU 247 (WDS 00095+1907) and BU 524 AB (WDS $02537+3820$ ) were revised, as announced previously in the IAU Commission 26 Information Circular Nos. 140 and 141, respectively.

All available micrometric and speckle measurements, together with the analytical method of Docobo (1985), were used to compute the orbits shown in Figs. 1 and 2. Speckle data are indicated by dots, while $\mathrm{O}-\mathrm{C}$ lines connect measures to their predicted locations on the orbit. For COU 247, visual measures are also included in the figure.

The solid line connects the primary component, indicated by the cross, with the periastron position. In Table 1 the individual orbital parameters are given for each star: line 1 - star identification; line 2 - orbit author(s); lines 3 to 9 - new orbital elements; line 10 - total mass of the system obtained by combining new $a$ and $P$ values with the Hipparcos parallax (ESA 1997). Because the COU 247 orbit may be evaluated only as preliminary, no errors for the individual elements are given in the table.

\section{COU 247}

The binarity of this star $(V=7.96$, sp.type G3V) was discovered by P. Couteau in 1967. Jasinta (1996) 
Table 2. Speckle measurements on the 1.52-m telescope (also available at the CDS via anonymous ftp cdsarc.u-strasbg.fr (130.79.128.5) or via http://cdsweb.u-strasbg.fr/cgi-bin/qcat?J/A+A/366/868)

\begin{tabular}{|c|c|c|c|c|c|c|}
\hline Coord. 2000 & Name/Catalog no. & $\begin{array}{l}\text { Discoverer } \\
\text { designation }\end{array}$ & $\begin{array}{l}\text { Epoch } \\
1999.0+ \\
\end{array}$ & $\begin{array}{c}\theta \\
\left({ }^{\circ}\right) \\
\end{array}$ & $\begin{array}{c}\rho \\
\left({ }^{\prime \prime}\right) \\
\end{array}$ & Note \\
\hline $00062+5826$ & ADS $61 \mathrm{AB}$ & STF 3062 & 0.7259 & 327.8 & 1.492 & \\
\hline $00095+1907$ & +180003 & COU 247 & 0.7118 & 308.7 & 0.301 & \\
\hline $00214+6700$ & ADS $293 \mathrm{AB}$ & STT 6 & 0.7232 & 154.2 & 0.622 & \\
\hline $00318+5431$ & $\mathrm{ADS} 434 \mathrm{AB}$ & STT 12 & 0.7231 & 196.6 & 0.382 & \\
\hline \multirow[t]{2}{*}{$00516+2237$} & ADS $701 \mathrm{AB}$ & A 1808 & 0.7119 & & & UR \\
\hline & & & 0.7230 & 197.5 & 0.165 & \\
\hline \multirow[t]{5}{*}{$00546+1911$} & ADS $746 \mathrm{AB}$ & STT 20 & 0.7229 & 194.7 & 0.526 & \\
\hline & & & 0.7229 & 194.3 & 0.519 & \\
\hline & & & 0.7254 & 194.2 & 0.516 & \\
\hline & & & 0.7254 & 194.1 & 0.523 & \\
\hline & & & 0.7280 & 194.2 & 0.518 & \\
\hline $00550+2338$ & ADS $755 \mathrm{AB}$ & STF 73 & 0.7256 & 308.2 & 0.884 & \\
\hline $00568+6022$ & ADS $784 \mathrm{AB}$ & BU 1099 & 0.7285 & 348.6 & 0.290 & \\
\hline $00583+2124$ & ADS $805 \mathrm{AB}$ & BU 302 & 0.7119 & 186.4 & 0.355 & \\
\hline 00594-0040 & ADS 819 AB & A 1902 & 0.7230 & 199.1 & 0.346 & \\
\hline \multirow[t]{2}{*}{$01030+4723$} & ADS $862 \mathrm{AB}$ & STT 21 & 0.7232 & & & UR \\
\hline & & & 0.7259 & 175.2 & 1.148 & \\
\hline \multirow[t]{7}{*}{$01040+3528$} & ADS $873 \mathrm{AB}$ & HO 213 & 0.7116 & 109.7 & 0.301 & \\
\hline & & & 0.7116 & 109.9 & 0.290 & $\mathrm{R}$ \\
\hline & & & 0.7116 & 109.3 & 0.301 & \\
\hline & & & 0.7119 & 108.8 & 0.302 & \\
\hline & & & 0.7120 & 110.3 & 0.303 & $\mathrm{R}$ \\
\hline & & & 0.7228 & & & UR \\
\hline & & & 0.7257 & 109.2 & 0.310 & \\
\hline $01049+3649$ & ADS $883 \mathrm{AB}$ & A 1515 & 0.7094 & 297.0 & 0.229 & \\
\hline $01148+6056$ & ADS 999 AB & BU 1100 & 0.7286 & & & UR \\
\hline \multirow[t]{2}{*}{$01178+4901$} & ADS $1040 \mathrm{AB}$ & STF 102 & 0.7230 & 276.2 & 0.482 & \\
\hline & & & 0.7285 & 275.6 & 0.480 & \\
\hline $01213+1132$ & ADS $1097 \mathrm{AB}$ & BU 4 & 0.7121 & 111.8 & 0.550 & \\
\hline \multirow[t]{2}{*}{$01234+5809$} & ADS $1105 \mathrm{AB}$ & STF 115 & 0.7259 & 177.6 & 0.166 & \\
\hline & & & 0.7285 & 176.7 & 0.166 & \\
\hline 01337-1213 & ADS $1223 \mathrm{AB}$ & HWE 4 & 0.7230 & & & UR \\
\hline \multirow[t]{2}{*}{ 01376-0924 } & -100343 & KUI 7 & 0.7228 & 149.0 & 0.288 & \\
\hline & & & 0.7258 & 148.9 & 0.282 & \\
\hline $01443+5732$ & ADS 1359 AB & BU 870 & 0.7286 & 347.2 & 0.726 & \\
\hline $01551+2847$ & ADS $1522 \mathrm{AB}$ & STF 183 & 0.7095 & 155.0 & 0.331 & \\
\hline \multirow[t]{2}{*}{$01559+0151$} & ADS 1538 AB & STF 186 & 0.7121 & 62.4 & 1.037 & \\
\hline & & & 0.7258 & 62.4 & 1.033 & \\
\hline $01570+3101$ & ADS $1548 \mathrm{AB}$ & A 819 & 0.7229 & & & UR \\
\hline $02039+4220$ & ADS $1630 \mathrm{BC}$ & STT 38 & 0.7258 & 105.1 & 0.477 & \\
\hline $02140+4729$ & ADS 1709 AB & STF 228 & 0.7096 & 282.5 & 1.007 & \\
\hline $02231+7021$ & +690144 & MLR 377 & 0.7286 & 145.3 & 0.659 & \\
\hline $02257+6133$ & ADS 1833 AB & STF 257 & 0.7286 & 64.9 & 0.390 & \\
\hline \multirow[t]{2}{*}{$02280+0158$} & +010431 & KUI 8 & 0.7228 & 36.5 & 0.520 & \\
\hline & & & 0.7258 & 37.2 & 0.516 & \\
\hline $02290+6724$ & ADS $1860 \mathrm{AB}$ & STF 262 & 0.7287 & 230.5 & 2.772 & \\
\hline $02407+2637$ & ADS 2034 AB & STT 43 & 0.7095 & 354.9 & 0.736 & \\
\hline \multirow[t]{3}{*}{$02537+3820$} & ADS $2200 \mathrm{AB}$ & STF 318 & 0.7095 & 5.9 & 0.156 & \\
\hline & & & 0.7095 & 5.3 & 0.159 & \\
\hline & & & 0.7121 & 5.6 & 0.162 & \\
\hline $02586+2408$ & ADS 2246 AB & BU 1173 & 0.7121 & 97.1 & 0.235 & \\
\hline $02589+2137$ & ADS 2253 AB & BU 525 & 0.7095 & 277.2 & 0.544 & \\
\hline \multirow[t]{2}{*}{$03054+2515$} & ADS 2336 AB & STF 346 & 0.7123 & 71.4 & 0.347 & \\
\hline & & & 0.7258 & 71.5 & 0.352 & \\
\hline $03096+0512$ & ADS $2373 \mathrm{AB}$ & A 2030 & 0.7124 & & & UR \\
\hline
\end{tabular}


Table 2. continued

\begin{tabular}{|c|c|c|c|c|c|c|}
\hline Coord. 2000 & Name/Catalog no. & $\begin{array}{l}\text { Discoverer } \\
\text { designation }\end{array}$ & $\begin{array}{l}\text { Epoch } \\
1999.0+\end{array}$ & $\begin{array}{c}\theta \\
\left({ }^{\circ}\right)\end{array}$ & $\begin{array}{c}\rho \\
\left({ }^{\prime \prime}\right)\end{array}$ & Note \\
\hline \multirow[t]{3}{*}{$03280+2028$} & ADS $2546 \mathrm{Aa}$ & COU 260 & 0.7231 & 24.9 & 0.245 & \\
\hline & & & 0.7231 & 24.2 & 0.252 & \\
\hline & & & 0.7288 & 24.9 & 0.247 & \\
\hline $03280+2028$ & ADS $2546 \mathrm{AB}$ & COU 260 & 0.7230 & 163.8 & 6.727 & \\
\hline \multirow[t]{2}{*}{$03284+6015$} & ADS 2538 AB & A 980 & 0.7124 & 353.1 & 0.349 & \\
\hline & & & 0.7124 & 351.8 & 0.344 & \\
\hline \multirow{2}{*}{$03503+2535$} & ADS 2799 & STT 65 & 0.7123 & 211.9 & 0.156 & \\
\hline & & & 0.7261 & 210.8 & 0.155 & \\
\hline $04263+3443$ & ADS $3211 \mathrm{AB}$ & HU 609 & 0.7260 & 328.7 & 0.220 & \\
\hline \multirow[t]{3}{*}{$04512+1104$} & ADS 3475 AB & BU 883 & 0.7234 & 77.4 & 0.236 & \\
\hline & & & 0.7260 & 78.4 & 0.237 & \\
\hline & & & 0.7260 & 79.1 & 0.228 & \\
\hline \multirow[t]{3}{*}{$05056+2304$} & +220818 & STT 97 & 0.7234 & 150.9 & 0.362 & $\mathrm{R}$ \\
\hline & & & 0.7260 & 150.5 & 0.359 & \\
\hline & & & 0.7260 & 149.9 & 0.357 & $\mathrm{R}$ \\
\hline $17146+1423$ & ADS $10418 \mathrm{AB}$ & STF 2140 & 0.7277 & 104.9 & 4.844 & \\
\hline \multirow{2}{*}{$17239-0050$} & ADS 10598 AB & STF 2173 & 0.7277 & 318.6 & 0.537 & \\
\hline & & & 0.7251 & & & UR \\
\hline $17563+0259$ & ADS 10899 AB & A 2189 & 0.7251 & & & UR \\
\hline $17575+1058$ & ADS 10916 AB & BU 1299 & 0.7251 & 83.8 & 0.244 & \\
\hline \multirow[t]{2}{*}{$17584+0428$} & +043562 & KUI 84 & 0.7250 & 85.0 & 0.231 & \\
\hline & & & 0.7250 & 84.3 & 0.227 & $\mathrm{R}$ \\
\hline 18031-0811 & ADS 11005 AB & STF 2262 & 0.7277 & 282.4 & 1.724 & \\
\hline $18055+0230$ & ADS 11046 AB & STF 2272 & 0.7277 & 149.2 & 3.685 & \\
\hline $18339+5221$ & ADS $11468 \mathrm{AB}$ & A 1377 & 0.7277 & 116.4 & 0.261 & \\
\hline $18355+2336$ & ADS 11479 AB & STT 359 & 0.7278 & 7.6 & 0.711 & \\
\hline $18386+1632$ & ADS $11530 \mathrm{AB}$ & HO 87 & 0.7251 & 72.1 & 0.365 & \\
\hline $18594-1250$ & -135172 & KUI 89 & 0.7114 & & & UR \\
\hline 19110-0726 & ADS $12126 \mathrm{AB}$ & A 95 & 0.7278 & 49.5 & 0.263 & \\
\hline \multirow[t]{7}{*}{$19159+2727$} & ADS $12239 \mathrm{AB}$ & STT 371 & 0.7250 & 160.3 & 0.888 & \\
\hline & & & 0.7252 & 160.1 & 0.885 & \\
\hline & & & 0.7252 & 160.2 & 0.890 & $\mathrm{R}$ \\
\hline & & & 0.7277 & 160.1 & 0.895 & $\mathrm{R}$ \\
\hline & & & 0.7277 & 159.0 & 0.883 & \\
\hline & & & 0.7278 & 160.0 & 0.880 & \\
\hline & & & 0.7280 & 160.2 & 0.883 & \\
\hline $19210+1909$ & ADS 12336 & STF 2504 & 0.7278 & 348.3 & 0.238 & \\
\hline $19459+5049$ & ADS 13135 & HU 687 & 0.7252 & & & UR \\
\hline $19487+1504$ & ADS 12961 & A 1658 & 0.7252 & 144.0 & 0.211 & \\
\hline $19487+3519$ & ADS $12972 \mathrm{AB}$ & STT 387 & 0.7279 & 141.4 & 0.618 & \\
\hline $19490+1909$ & ADS $12973 \mathrm{AB}$ & AGC 11 & 0.7278 & 141.0 & 0.153 & \\
\hline $20203+3924$ & ADS $13728 \mathrm{AB}$ & A 1427 & 0.7279 & 118.7 & 0.310 & \\
\hline $20375+1436$ & ADS $14073 \mathrm{AB}$ & BU 151 & 0.7279 & 340.5 & 0.498 & \\
\hline \multirow[t]{2}{*}{$20396+0458$} & +044510 & KUI 99 & 0.7115 & 126.9 & 0.315 & \\
\hline & & & 0.7115 & 126.5 & 0.324 & \\
\hline $20537+5918$ & ADS 14412 AB & A 751 & 0.7279 & & & UR \\
\hline $21001+0731$ & +064718 & KUI 102 & 0.7279 & 11.9 & 0.346 & \\
\hline $21208+3227$ & ADS $14889 \mathrm{AB}$ & STT 437 & 0.7280 & 22.5 & 2.348 & \\
\hline $21441+2845$ & ADS $15270 \mathrm{AB}$ & STF 2822 & 0.7255 & 307.3 & 1.915 & \\
\hline $21501+1717$ & +164612 & COU 14 & 0.7256 & 246.5 & 0.338 & \\
\hline $21597+4907$ & ADS $15530 \mathrm{AB}$ & HU 774 & 0.7280 & 347.7 & 0.192 & \\
\hline $22241-0450$ & ADS $15902 \mathrm{AB}$ & BU 172 & 0.7116 & 63.1 & 0.288 & \\
\hline 22288-0001 & ADS $15971 \mathrm{AB}$ & STF 2909 & 0.7255 & 188.2 & 1.919 & \\
\hline $22302+2228$ & ADS $15992 \mathrm{AB}$ & HU 388 & 0.7255 & 236.8 & 0.490 & \\
\hline $22307+1758$ & +174759 & COU 234 & 0.7256 & & & UR \\
\hline
\end{tabular}


Table 2. continued

\begin{tabular}{llllrl}
\hline Coord. 2000 & Name/Catalog no. & $\begin{array}{l}\text { Discoverer } \\
\text { designation }\end{array}$ & $\begin{array}{l}\text { Epoch } \\
1999.0+\end{array}$ & $\begin{array}{r}\theta \\
\left({ }^{\circ}\right)\end{array}$ & $\begin{array}{c}\rho \\
\left(^{\prime \prime}\right)\end{array}$ \\
\hline $22402+3732$ & ADS 16164 AB & HO 188 & 0.7280 & 215.7 & 0.372 \\
$23114+3813$ & ADS 16576 AB & HO 197 & 0.7282 & 302.4 & 0.288 \\
$23126+0241$ & ADS 16591 AB & A 2298 & 0.7282 & 296.9 & 0.235 \\
$23176+1818$ & ADS 16650 AB & HU 400 & 0.7120 & 101.6 & 0.343 \\
$23340+3120$ & ADS 16836 AB & BU 720 & 0.7282 & 94.2 & 0.550 \\
& & & 0.7255 & 94.4 & 0.546 \\
$23393+4543$ & ADS 16904 AB & A 643 & 0.7282 & 150.2 & 0.239 \\
$23440+2922$ & ADS 16957 AB & AGC 14 & 0.7117 & 87.7 & 0.837 \\
& & & 0.7120 & 88.0 & 0.827 \\
$23475+4650$ & ADS 17006 AB & BU 995 & 0.7118 & 246.1 & 0.768 \\
$23561+2520$ & ADS 17105 AB & A 426 & 0.7117 & 301.7 & 0.394 \\
& & & 0.7117 & 304.2 & 0.385 \\
$23595+5441$ & ADS 17151 AB & A 1498 & 0.7283 & 88.1 & 0.378 \\
\hline
\end{tabular}

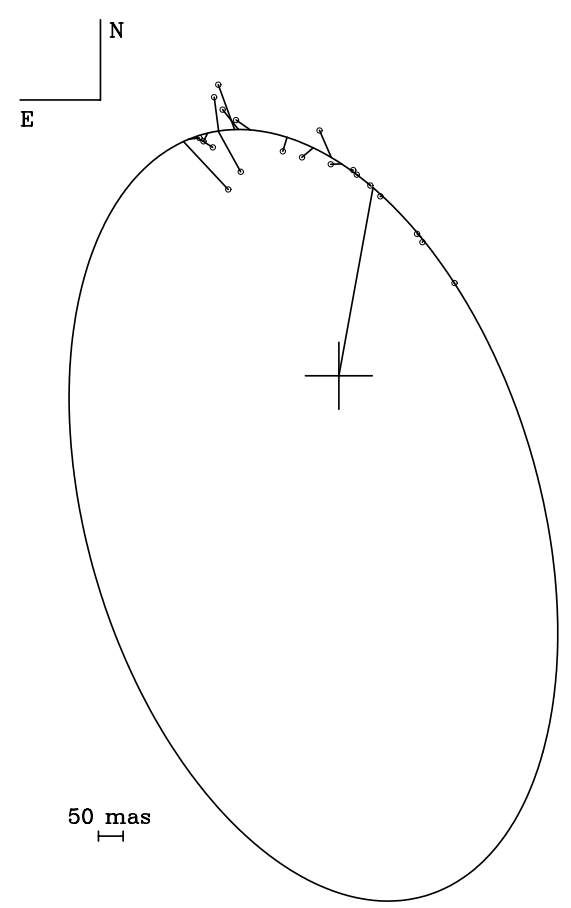

Fig. 1. Preliminary orbit for COU 247

calculated the orbit with a period of 60.52 years. However, new speckle measurements indicate a longer period. Although the binary has completed only about $80^{\circ}$ of orbital motion, the new orbital solution gives the dynamical parallax (15 mas), which is in agreement with the Hipparcos parallax value (13.8 mas).

\section{BU $524 \mathrm{AB}$}

Due to its short period, this bright binary star $(V=5.36$, sp. type F4IV) has completed several revolutions since the discovery of its binary nature by S. W. Burnham in 1878 . Nevertheless, the last orbit, calculated for the system by Aristidi et al. (1999), does not fit well with recent speckle measurements. We tried to adjust the orbital parameters to the latest speckle data, and with these improvements, the orbit seems to be almost definitive.



Fig. 2. New visual orbit for BU $524 \mathrm{AB}$

Acknowledgements. The development of the speckle camera was entirely financed by the Secretaria Xeral de Investigacion e Desenvolvemento of the Xunta de Galicia (regional government of Galicia, Spain) on the basis of the Call for Proposals for Science Equipment in 1998. This work is a part of the Investigation Project PGIDT99PXI24301B supported by the Xunta de Galicia.

Authors thank Observatorio Astronomico Nacional for facilities made available at Calar Alto and Dr. V. Lanchares and P. Abelleira for their assistance with the observations.

\section{References}

Aristidi, E., Prieur, J. L., Scardia, M., et al. 1999, A\&AS, 134, 545

Docobo, J. A. 1985, Celestial Mechan., 36, 143

Douglass, G. G., Hindsley, R. B., \& Worley, C. E. 1997, AJ, 111,289

ESA, 1997, The Hipparcos and Tycho catalogues (ESA SP1200)

Jasinta, D. M. D. 1996, A\&AS, 118, 381

Labeyrie, A. 1970, A\&A, 6, 85

Worley, C. E., \& Douglass, G. G. 1996, The Washington Double Star Catalog (WDS),

http://aries.usno.navy.mil/ad/wds/ 